\title{
Coordination of Fast and Slow Rhythmic Neuronal Circuits
}

\author{
Marlene Bartos,, ${ }^{1}$ Yair Manor, ${ }^{2}$ Farzan Nadim, ${ }^{2}$ Eve Marder, $^{2}$ and Michael P. Nusbaum 1 \\ ${ }^{1}$ Department of Neuroscience, University of Pennsylvania School of Medicine, Philadelphia, Pennsylvania 19104-6074, \\ and 2 Volen Center for Complex Systems, Brandeis University, Waltham, Massachusetts 02454
}

Interactions among rhythmically active neuronal circuits that oscillate at different frequencies are important for generating complex behaviors, yet little is known about the underlying cellular mechanisms. We addressed this issue in the crab stomatogastric ganglion (STG), which contains two distinct but interacting circuits. These circuits generate the gastric mill rhythm (cycle period, $\sim 10 \mathrm{sec}$ ) and the pyloric rhythm (cycle period, $\sim 1 \mathrm{sec}$ ). When the identified modulatory projection neuron named modulatory commissural neuron 1 (MCN1) is activated, the gastric mill motor pattern is generated by interactions among MCN1 and two STG neurons [the lateral gastric (LG) neuron and interneuron 1]. We show that, during MCN1 stimulation, an identified synapse from the pyloric circuit onto the gastric mill circuit is pivotal for determining the gastric mill cycle period and the gastric-pyloric rhythm coordination. To examine the role of this intercircuit synapse, we replaced it with a computational equivalent via the dynamic-clamp technique. This enabled us to manipulate better the timing and strength of this synapse. We found this synapse to be necessary for production of the normal gastric mill cycle period. The synapse acts, during each LG neuron interburst, to boost rhythmically the influence of the modulatory input from MCN1 to LG and thereby to hasten LG neuron burst onset. The two rhythms become coordinated because LG burst onset occurs with a constant latency after the onset of the triggering pyloric input. These results indicate that intercircuit synapses can enable an oscillatory circuit to control the speed of a slower oscillatory circuit, as well as provide a mechanism for intercircuit coordination.

Key words: stomatogastric ganglion; neuromodulation; intercircuit coordination; disinhibition; pyloric rhythm; gastric mill rhythm; modulatory projection neuron
The CNS contains oscillatory networks that generate a wide variety of rhythms. Some of these networks, such as those that generate rhythmic motor patterns, are clearly associated with behavior (Marder and Calabrese, 1996). Other CNS oscillations are posited to play roles in sensory processing (Gray, 1995; Laurent, 1996, 1997), sleep and arousal (McCormick and Bal, 1997), and learning (Lisman, 1997). Additionally, widespread synchronous oscillatory activity in the brain can be pathological (McCormick and Bal, 1997). As we work to understand how these oscillatory networks contribute to behavior, it is important to understand how they interact. Much of our intuition about how oscillators interact comes from systems such as the heart, in which the component elements are similar in properties and in period. Segmentally iterated oscillatory circuits, such as those underlying locomotion, have also received considerable attention. However, a cellular level understanding of their intercircuit interactions is

Received March 15, 1999; revised May 13, 1999; accepted May 13, 1999.

This research was supported by National Institute of Neurological Disorders and Stroke Grants NS-29436 (M.P.N.) and NS-17813 (E.M.), the German Science foundation (Deutsche Forschungsgemeinschaft; M.B.), the United States-Israel Binational Science Foundation (Y.M.; M.P.N.), the Sloan Center for Theoretical Biology at Brandeis University, and the W. M. Keck Foundation. We thank Dr. Dawn M. Blitz for providing constructive criticism to previous versions of this paper and Dr. Jorge Golowasch for helpful discussions.

Correspondence should be addressed to Dr. Michael P. Nusbaum, Department of Neuroscience, University of Pennsylvania School of Medicine, 215 Stemmler Hall, Philadelphia, PA 19104-6074.

Dr. Bartos' present address: University of Freiburg, Physiology 1, D-79104 Freiburg, Germany.

Dr. Manor's present address: Life Sciences Department, Ben-Gurion University of the Negev, Beer-Sheva, Israel 84105.

Dr. Nadim's present address: Department of Mathematical Sciences, New Jersey Institute of Technology, and Federated Department of Biological Sciences, Rutgers University, Newark, NJ 07102.

Copyright (C) 1999 Society for Neuroscience $0270-6474 / 99 / 196650-11 \$ 05.00 / 0$ not available (Skinner and Mulloney, 1998). Significantly less is understood about the interactions between neuronal or network oscillators that generate activity patterns with significantly different cycle periods.

The stomatogastric nervous system of the crab Cancer borealis can be used effectively to explore the mechanisms by which the output of a network oscillator is controlled by a distinct but behaviorally related oscillatory circuit. In $C$. borealis, overlapping subsets of neurons in the stomatogastric ganglion (STG) compose circuits that generate the gastric mill (cycle period, $\sim 10 \mathrm{sec}$ ) and pyloric (cycle period, $\sim 1 \mathrm{sec}$ ) rhythms (Weimann et al., 1991; Weimann and Marder, 1994). These circuits control chewing and the filtering of chewed food, respectively (Harris-Warrick et al., 1992). The STG receives extensive modulatory input, primarily from the neighboring commissural ganglia, that enables the generation of many versions of the gastric mill and pyloric rhythms (Coleman et al., 1992; Marder and Calabrese, 1996; Marder et al., 1997a). One well-characterized modulatory input to the STG is modulatory commissural neuron 1 (MCN1) (Nusbaum et al., 1992; Coleman and Nusbaum, 1994). Activation of MCN1 elicits the gastric mill rhythm and strengthens the ongoing pyloric rhythm (Coleman and Nusbaum, 1994; Coleman et al., 1995; Bartos and Nusbaum, 1997a).

The experimental work presented here was motivated by a previous modeling study that predicted that, during MCN1 stimulation, an identified synapse from the pyloric circuit onto the gastric mill circuit was necessary to generate the normal gastric mill cycle period and to coordinate the activity of these two rhythms (Nadim et al., 1998). Here, we exploit the dynamic-clamp technique (Sharp et al., 1993a,b) to replace this identified synapse with an artificial conductance that simulates the synapse. This 
allows us to vary systematically its strength and timing and to assess their influence on the gastric mill rhythm. We verify many of the predictions of the computational model, although the absolute requirement of pyloric inhibition for the expression of the gastric mill rhythm was not found.

Parts of this paper have been published previously in abstract form (Bartos and Nusbaum, 1997b; Manor et al., 1998).

\section{MATERIALS AND METHODS}

Animals. Crabs (C. borealis) were obtained from commercial suppliers (Boston, MA) and the Marine Biological Laboratory (Woods Hole, MA). Animals were maintained in aerated artificial seawater at $10-12^{\circ} \mathrm{C}$ and were cold anesthetized by packing in ice for 20-40 min before dissection. The stomach, including the stomatogastric nervous system, was removed from the animal, and the rest of the dissection was performed in chilled $\left(\sim 4^{\circ} \mathrm{C}\right)$ physiological saline. Data were obtained from 56 male crabs.

Solutions. C. borealis physiological saline had the following composition (in mM): $\mathrm{NaCl}, 440 ; \mathrm{MgCl}_{2}, 26 ; \mathrm{CaCl}_{2}, 13 ; \mathrm{KCl}, 11$; Trizma base, 10; and maleic acid, 5, pH 7.4-7.6.

Electrophysiology. Electrophysiological experiments were performed using standard techniques for this system (Bartos and Nusbaum, 1997a). The isolated stomatogastric nervous system (Fig. 1) was pinned down in a silicone elastomer (SYLGARD 184: KR Anderson, Santa Clara, CA)lined Petri dish. All preparations were superfused continuously with $C$. borealis physiological saline $\left(10-13^{\circ} \mathrm{C}\right)$. Extracellular recordings were made by pressing stainless steel pin electrodes into the SYLGARD alongside the nerves and isolating each area with petroleum jelly (Vaseline; Chesebrough-Ponds, Greenwich, CT). The desheathed ganglia were viewed with light transmitted through a dark-field condenser (Nikon) to facilitate intracellular recordings. Intracellular recordings of STG somata were made using microelectrodes (15-30 M $\Omega$ ) filled with $4 \mathrm{M}$ potassium acetate and $20 \mathrm{~mm}$ potassium chloride.

The stomatogastric nervous system includes the paired commissural ganglia, the oesophageal ganglion, and the STG (Fig. 1A). All experiments were performed in the isolated stomatogastric nervous system after both inferior oesophageal nerves (ions) and superior oesophageal nerves (sons) were transected. MCN1 was selectively stimulated extracellularly via the ions $(10-20 \mathrm{~Hz})$ to activate the gastric mill rhythm (Fig. 1B) (Coleman et al., 1995; Bartos and Nusbaum, 1997a). The ions were stimulated using a Grass S88 stimulator and Grass SIU5 stimulus isolation unit (Astro-Med/Grass Instruments, Warwick, RI). One gastric mill cycle was defined as the duration between the onset of an impulse burst in the lateral gastric (LG) neuron and the onset of the subsequent LG neuron burst. A pyloric cycle was defined as extending from the onset of one pyloric dilator (PD) neuron burst to the onset of the subsequent PD neuron burst. STG neurons were identified on the basis of their axonal projections, their activity patterns, and their interactions with other STG neurons (Weimann et al., 1991; Norris et al., 1994, 1996; Bartos and Nusbaum, 1997a). Data were collected on a chart recorder (Astro-Med/ Grass Instruments) and videotape (Vetter Instruments, Rebersburg, PA).

Dynamic clamp. The dynamic-clamp technique (Sharp et al., 1993a,b) was used to create an artificial synapse that replaced the influence of the anterior burster (AB) neuron on the gastric mill circuit. This technique is used to add an artificial ionic conductance into a neuron using a computer and an intracellular microelectrode. The dynamic-clamp program continually monitors the membrane potential of the neuron to be influenced, via input from the intracellular recording amplifier. All such recordings were performed in single-electrode discontinuous current clamp (2-3 kHz sample rate; Axoclamp 2; Axon Instruments). The amount of current injected into the neuron is dynamically determined as $I=g\left(E_{\mathrm{rev}}-\mathrm{V}_{m}\right)$, where $\mathrm{V}_{m}$ is the cell membrane potential at the time of injection and $g$ and $E_{\text {rev }}$ are predetermined values for the conductance and the reversal potential, respectively, of the artificial ionic current. If the conductance to be modeled is voltage- and time-dependent, then $g$ is calculated according to the appropriate differential equations describing those properties. Unlike direct current injection, the dynamic-clamp current affects the input resistance of the cell, just as is done by a biological conductance (Sharp et al., 1993a,b).

For the experiments described here, the dynamic-clamp method was modified and implemented using software written by two of us (Y.M. and
F.N.) in LabWindows/CVI for an AT-MIO-16E2 analog-to-digital board (National Instruments, Houston, TX). The artificial pyloric-like clock was kept independent of gastric mill activity. In some experiments, pyloric-like dynamic-clamp pulses were injected into interneuron 1 (Int1) during the Int1 bursts. In other experiments, these pulses were injected into the LG neuron during the LG interbursts. LG does not receive the biological pyloric-timed input during the LG burst because it is inhibiting the source of this input (Int1). On the basis of electrophysiological measurements, the reversal potential of both the artificial inhibitory synapse injected into Int1 and the disinhibition injected into LG was set at $-80 \mathrm{mV}$. The dynamic-clamp synaptic current had a half-sine waveform and a duration in the physiological range $(250-600 \mathrm{msec})$. These waveforms approximate the effect of graded synaptic release (Graubard et al., 1980; Johnson and Harris-Warrick, 1990; Manor et al., 1997) from the AB neuron. Statistical analyses were performed using SigmaStat, version 2.0 (SPSS, Chicago, IL).

\section{RESULTS \\ MCN1-elicited gastric mill rhythm generation}

In $C$. borealis, there is generally a relatively slow pyloric rhythm and no gastric mill rhythm when the ions and sons are transected. This is evident in Figure $1 B$ in which, before MCN1 stimulation, the pyloric rhythm was present but the gastric mill rhythm was silent. The pyloric rhythm is represented by the activity of the AB neuron. Although Int1 is part of the gastric mill system, when the gastric mill rhythm is not active, Int1 fires in pyloric time because it is inhibited by the AB neuron. When MCN1 was stimulated, the gastric mill rhythm was activated, and the LG neuron fired alternating bursts with Int1 and the dorsal gastric (DG) neuron. There is also a slow excitation of the pyloric pacemaker ensemble, including the $\mathrm{AB}$ neuron and the PD neurons, by MCN1 (Bartos and Nusbaum, 1997a).

Figure $1 C$ shows the connectivity that gives rise to the gastric mill rhythm produced in Figure $1 B$. The core of the network is the reciprocal inhibition between the LG neuron and Int1. MCN1 produces a slow excitation of the LG and DG neurons and a fast chemical excitatory synapse onto Int1 (Coleman and Nusbaum, 1994; Coleman et al., 1995). A critical feature of the operation of this circuit is the presynaptic inhibition of the MCN1 terminals by the LG neuron (Nusbaum et al., 1992; Coleman and Nusbaum, 1994). This means that when the LG neuron is active, the terminals of MCN1 are inhibited and no longer release neurotransmitter. This action converts a tonic modulatory input into an intermittent excitatory drive (Coleman and Nusbaum, 1994; Coleman et al., 1995). The influence of the modulatory input to LG thus wanes during the LG burst and then must build up again during each subsequent LG interburst.

Figure $1 B$ shows another important feature of the activation of the gastric mill rhythm by MCN1. The arrow shows the onset of fast pyloric-timed depolarizations in the LG neuron that are present during each LG interburst. These depolarizations are caused by the periodic removal (disinhibition) of the Int1-evoked inhibition of the LG neuron. This disinhibition is a result of the rhythmic inhibition of Int1 by the AB neuron. These rhythmic depolarizations grow in amplitude because the MCN1-mediated slow depolarization of the LG neuron moves it further from the reversal potential of the Int1 to LG synapse.

Initially it was thought that the strength and time course of the MCN1 input were the most critical determinants of the gastric mill rhythm cycle period (Coleman et al., 1995). On the basis of the data of Coleman et al. (1995), Nadim et al. (1998) developed 

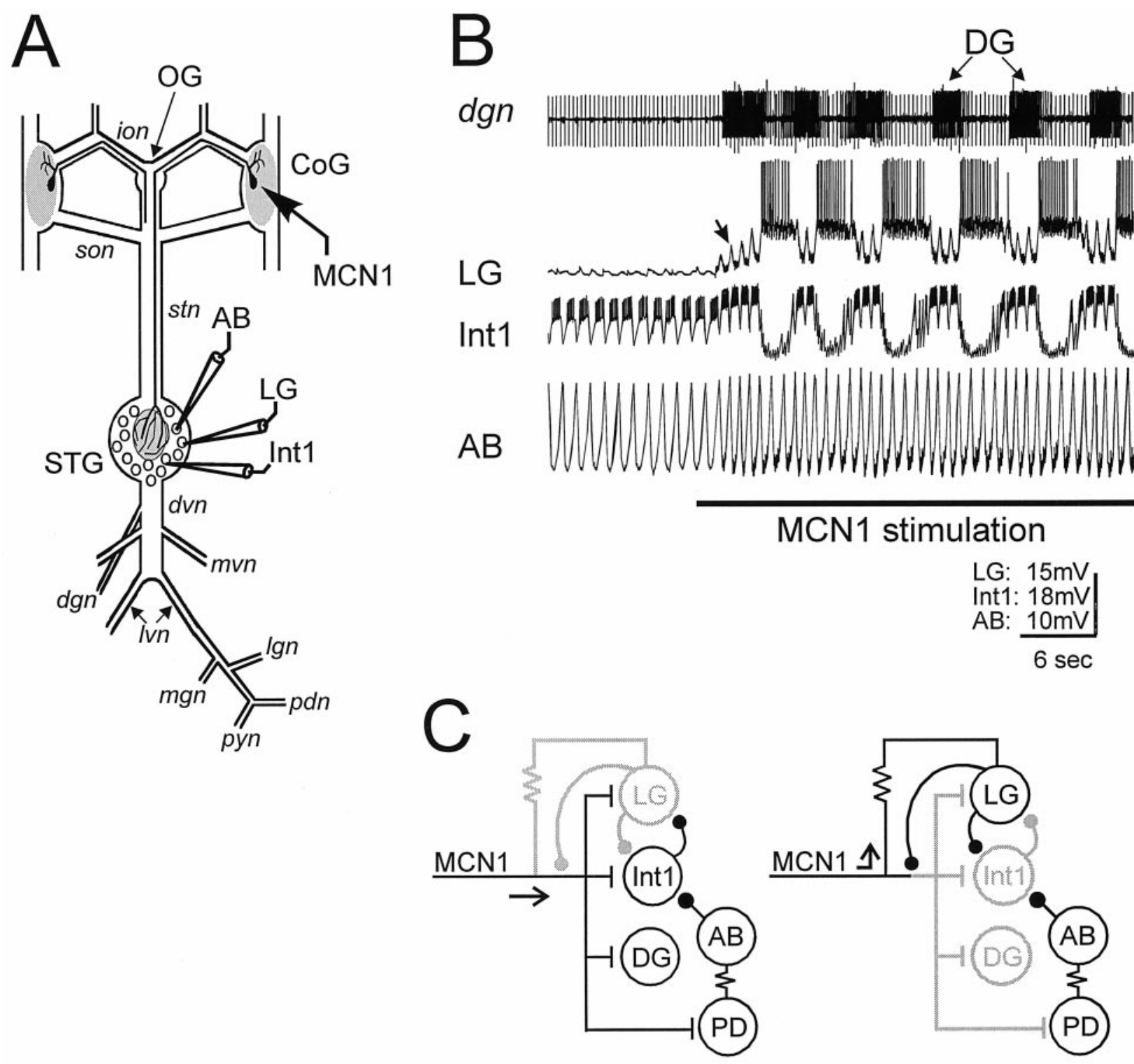

Figure 1. Activation of $M C N 1$ elicits a gastric mill rhythm in the $S T G$. A, Schematic illustration of the isolated stomatogastric nervous system, including the soma location and axonal projection pathway of $M C N 1$. B. Activation of the gastric mill rhythm in the isolated $S T G$ by stimulation of $M C N 1$. Before $M C N 1$ stimulation, there was no gastric mill rhythm ( $L G$ and $D G$ were silent; Int1 fired pyloric-timed bursts), but there was an ongoing pyloric rhythm ( $A B$ neuron recording). During tonic stimulation of both MCN1 neurons (extracellular stimulation of both ions at $10 \mathrm{~Hz}$ each) (Bartos and Nusbaum, 1997a), the pyloric rhythm cycled faster, and the gastric mill rhythm was activated. The pyloric-timed subthreshold oscillations in $L G$ during $M C N 1$ stimulation result from pyloric-timed inhibition of Int1, producing rhythmic disinhibitions in $L G$ (arrow). The smaller amplitude, bursting unit in the dgn recording is the $D G$ neuron (arrows), whereas the tonically firing unit is the anterior gastric receptor (AGR), an identified sensory neuron. Most hyperpolarized $\mathrm{V}_{m}$ values: $A B,-60 \mathrm{mV}$; Int $1,-56 \mathrm{mV}$; and $L G,-72 \mathrm{mV}$. C, Schematic circuit diagram underlying $M C N 1$ activation of the gastric mill rhythm. The circuit represents the two phases of the gastric mill rhythm, including retraction (left; Int 1 and $D G$ active) and protraction (right; $L G$ active). Members of the pyloric pacemaker ensemble, the $A B$ and $P D$ neurons, are also included. The $M C N 1$ synapse on $P D$ is a functional representation. It is not determined whether $M C N 1$ directly excites $P D, A B$, or both neurons. Arrows represent the pathway of functioning $M C N 1$ transmission. Active neurons and synapses are labeled black, whereas inactive neurons and synapses are labeled gray. T-bars represent excitatory chemical transmission; filled circles represent inhibitory chemical transmission; and resistor symbols represent electrical transmission. Nerves: $d v n$, dorsal ventricular nerve; $d g n$, dorsal gastric nerve; ion, inferior oesophageal nerve; lgn, lateral gastric nerve; lvn, lateral ventricular nerve; $m g n$, medial gastric nerve; $m v n$, medial ventricular nerve; $p d n$, pyloric dilator nerve; pyn, pyloric nerve; son, superior oesophageal nerve; stn, stomatogastric nerve. Ganglia: $C o G$, commissural ganglion; $O G$, oesophageal ganglion; $S T G$, stomatogastric ganglion. Neurons: $A B$, anterior burster; $D G$, dorsal gastric; Int1, interneuron 1; $L G$, lateral gastric; $M C N 1$, modulatory commissural neuron $1 ; P D$, pyloric dilator. Subsets of these abbreviations also appear on subsequent figures.

a computational model that reproduced the MCN1-elicited gastric mill rhythm. This model predicted that the period of the gastric mill rhythm is indeed influenced by the strength of MCN1 activity, but it also predicted that the gastric mill cycle period is influenced by the strength and frequency of the inhibitory syn- apse from $\mathrm{AB}$ to Int1 (Fig. 1C). Despite years of study of the motor patterns in the STG, the importance of the inhibitory synapse from AB to Int1 had not been appreciated. We therefore tested this prediction by comparing MCN1-elicited gastric mill rhythms with and without an accompanying pyloric rhythm. 

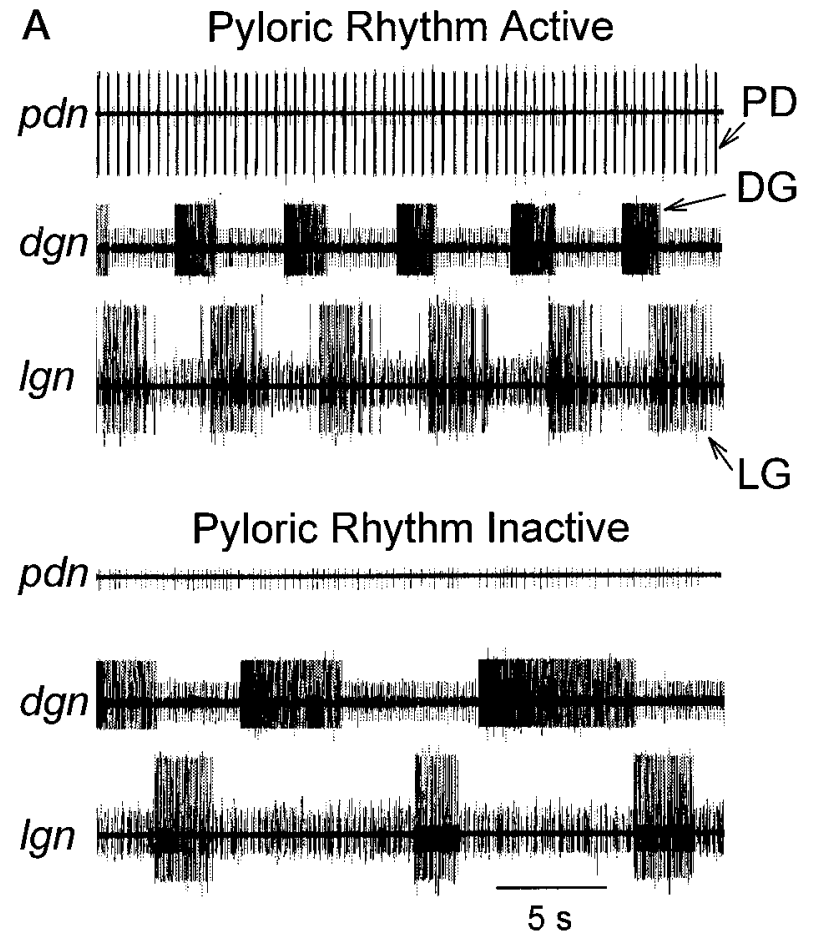

Influence of the pyloric rhythm and MCN1 on the gastric mill rhythm cycle period

We began testing the predictions of the Nadim et al. (1998) model by examining the gastric mill system response to MCN1 stimulation in the absence of a pyloric rhythm. The pyloric rhythm was turned off by injecting hyperpolarizing current into the AB neuron and/or its electrically coupled partners, the PD neurons (Figs. $1 C, 2 A)$. In 22 of 24 preparations in which the pyloric rhythm was stopped, MCN1 activation still elicited a gastric mill rhythm, but this rhythm was significantly slower than that when the pyloric rhythm was active (Fig. 2). The mean gastric mill cycle period increased from $7.1 \pm 2.8$ to $19.1 \pm 6.0 \mathrm{sec}(p \leq 0.001$, MannWhitney test; $n=22$ ). In the remaining two preparations, this treatment eliminated the gastric mill rhythm (data not shown). When the pyloric rhythm is shut off, the fast rhythmic inhibition from AB to Int1 is eliminated, as is the fast rhythmic disinhibition of LG. During an ongoing pyloric rhythm, these pyloric-timed disinhibitions in LG grow steadily in amplitude during any individual LG interburst interval, until one of these events triggers an LG burst (Nadim et al., 1998) (see Fig. $1 B$ and below). On the basis of previous physiological data (Coleman et al., 1995) and the predictions of Nadim et al. (1998), we hypothesized that these pyloric-timed inputs to the gastric mill system act in concert with the slow modulatory excitation from MCN1 to reduce the time needed by LG to escape from Int1 inhibition and fire its next impulse burst. We therefore tested the contributions of these two inputs to the determination of the gastric mill cycle period.

First, we examined the extent to which the gastric mill period was influenced by the level of input from MCN1. To this end, we stimulated MCN1 to fire tonically at different frequencies. Whether the pyloric rhythm was active (Fig. $3 A$, left ) or not active (Fig. $3 A$, right), increasing levels of MCN1 activity consistently elicited faster gastric mill rhythms (two-way ANOVA, $p<0.001$ ). Note, however, that at any given MCN1-firing frequency, the gastric mill rhythm was always slower when the pyloric rhythm
Figure 2. The pyloric rhythm regulates the cycle period of the gastric mill rhythm. $A$, Top, In the isolated STG, MCN1 stimulation (both ions at $10 \mathrm{~Hz}$ each; data not shown) drives a vigorous pyloric rhythm $(p d n)$ and gastric mill rhythm, represented by the alternating bursts in $D G(\operatorname{dgn} n)$ and $L G(\lg n)$. Bottom, In the same preparation, after the pyloric rhythm was turned off by injecting hyperpolarizing current into the pyloric pacemaker neurons (note the absence of bursting in $p d n$ ), the same level of MCN1 stimulation elicited a slower gastric mill rhythm. $B$, Histograms representing the mean gastric mill cycle period during MCN1 stimulation in the presence (pyloric rhythm on, $7.1 \pm 2.8$ sec; $n=22$ ) and absence (pyloric rhythm off, $19.1 \pm 6.0 \mathrm{sec} ; n=22$ ) of the pyloric rhythm are shown. The gastric mill cycle period was longer when the pyloric rhythm was off (Mann-Whitney test, $\left.{ }^{* *} p<0.001\right)$.
Active Inactive (Control) Pyloric Rhythm was off (two-way ANOVA, $p<0.001$; Fig. 3B). Similar results were obtained across several experiments $(n=6)$.

\section{The $A B$ neuron to Int1 synapse contributes to gastric mill cycle period regulation}

To understand the mechanism of the $\mathrm{AB}$ influence on the gastric mill rhythm, we wished to eliminate the effect of the biological $\mathrm{AB}$ and to replace it with a pyloric-timed synaptic conductance that we could control. Therefore we used the dynamic-clamp technique (Sharp et al., 1993a,b; Manor and Nadim, 1997) to create an artificial AB to Int1-like synapse (see Materials and Methods). After the pyloric rhythm was eliminated (by hyperpolarizing $\mathrm{AB}$ ), we added pyloric-like dynamic-clamp inhibitory artificial synaptic potentials into Int1. We kept the frequency of the pyloric-like pulses fixed at a period of $1 \mathrm{sec}$, which commonly occurs during MCN1 activation (Bartos and Nusbaum, 1997a). We also maintained a constant MCN1-firing frequency. Figure 4 shows the rhythmic alternations between LG and Int1 that resulted from dynamic-clamp injections of pyloric-like pulses into Int1. Each AB-like hyperpolarization of Int1 produced a disinhibition in LG, similar to those occurring during natural pyloric rhythms (see Figs. $1 B, 3 A$ ). Note the absence of these rhythmic disinhibitions in LG when the dynamic clamp was not activated and the pyloric rhythm was off (Fig. 4). Providing the pyloric-like pulses also decreased the gastric mill cycle period. Recordings of several additional members of the gastric mill system, including DG and the medial gastric (MG) neuron, are also shown. These additional gastric mill neurons have little influence on gastric mill pattern generation and are primarily motor neurons. With neither the pyloric rhythm nor the dynamic-clamp active, the firing pattern of one or more of these gastric mill neurons is often altered from the pattern during a normal MCN1-elicited gastric mill rhythm (Fig. 4) (Coleman and Nusbaum, 1994; Coleman et al., 1995). For example, in Figure 4, DG neuron activity was less regular, and the MG neuron exhibited no activity during the LG interburst. Activation of the dynamic-clamp injections made the 

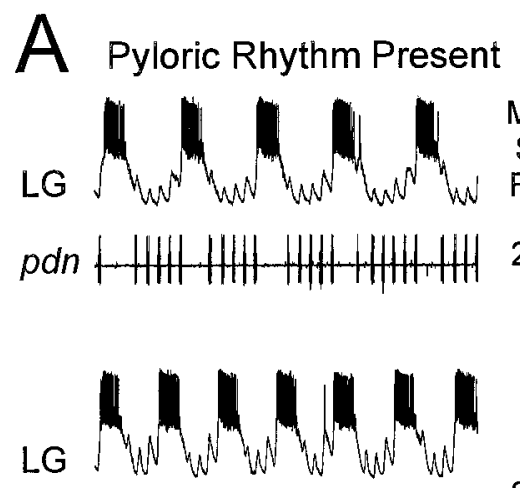

pdn HHHHHH+HHHHHHHHHHHH

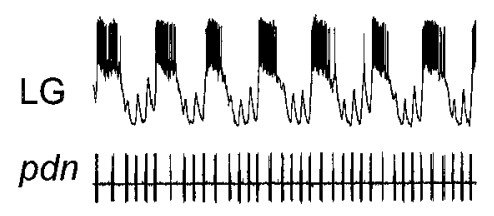

$25 \mathrm{~Hz}$

Pyloric Rhythm Absent

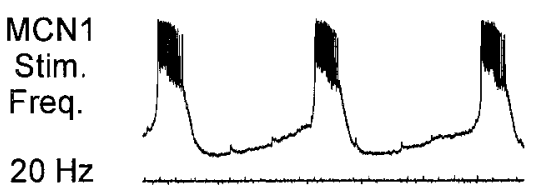

$30 \mathrm{~Hz}$
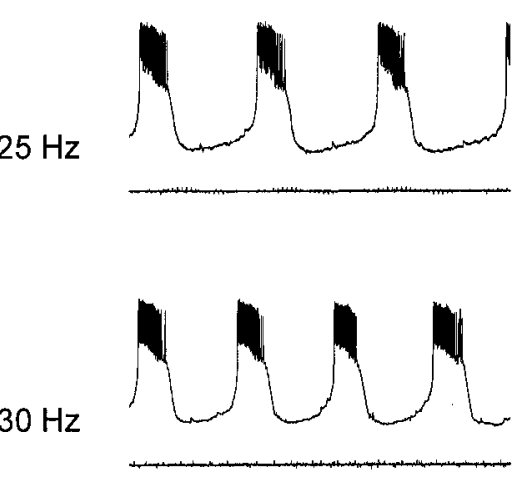

B
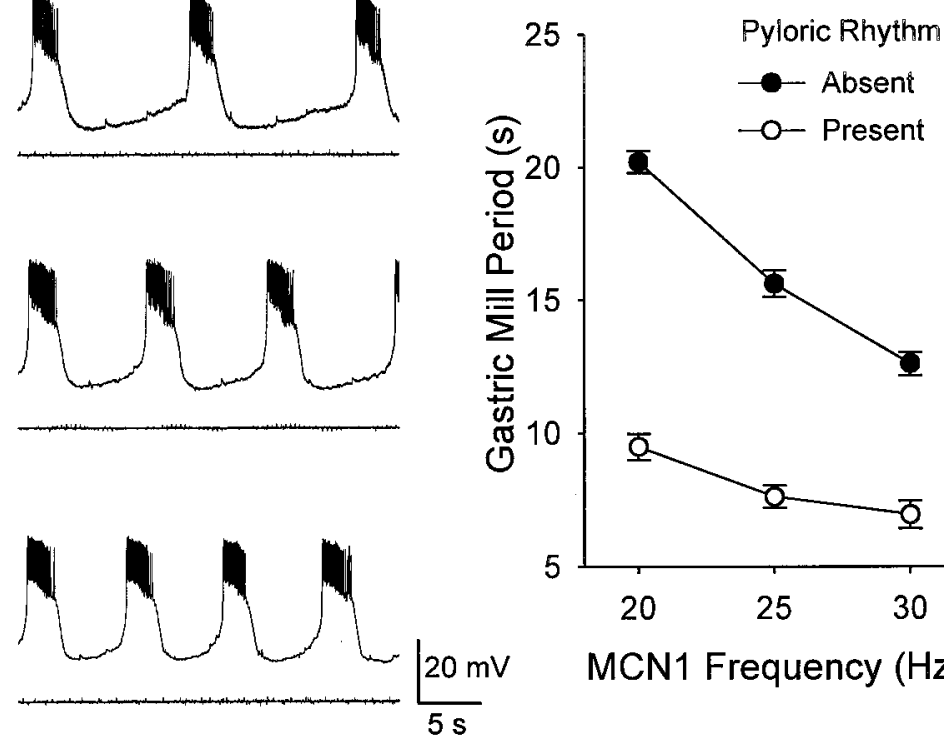

-O- Present
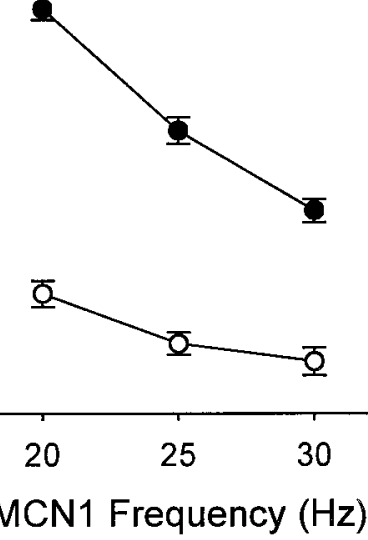

Figure 3. The gastric mill cycle period is a function of $M C N 1$-firing frequency. A, Left, With the pyloric rhythm in progress, increasing the $M C N 1$-firing frequency decreases the gastric mill cycle period. Most hyperpolarized $\mathrm{V}_{m}: L G,-66 \mathrm{mV}$. Right, When the pyloric rhythm is turned off by hyperpolarization of the pyloric pacemaker neurons, the gastric mill cycle period still decreases with increasing levels of $M C N 1$ activity. Note, however, that at any level of $M C N 1$ activity the gastric mill cycle period is shorter when the pyloric rhythm is present. Each indicated $M C N 1$-firing frequency represents its activity across left and right panels. Most hyperpolarized $\mathrm{V}_{m}: L G,-74 \mathrm{mV}$. B. Plot of the gastric mill period (mean $\pm \sigma$ ) as a function of MCN1 frequency in the presence and absence of the pyloric rhythm is shown. Stim. Freq., Stimulation frequency.

activity of these neurons comparable with that occurring during the normal MCN1-elicited gastric mill rhythm. Figure 4 therefore demonstrates that the dynamic-clamp substitution for the natural pyloric input is sufficient to produce a representative gastric mill motor pattern.

The model by Nadim et al. (1998) predicted that the strength and period of the pyloric input contribute in different ways to gastric mill rhythm generation. The strength of this input was predicted to influence the gastric mill period by influencing the amplitude of the disinhibition in LG. The function of the periodicity of this input was predicted to be to place a single disinhibition as close in time as possible to the time when LG first becomes capable of generating a burst, after the buildup of modulatory input from MCN1. Experimental separation of these two parameters by directly manipulating the $\mathrm{AB}$ neuron is not possible. This is because, under the latter condition, changing either parameter alters the other one. However, the dynamicclamp technique enabled us to obtain a reconstituted gastric mill motor pattern in which the period and strength of the pyloric input could be manipulated separately and each effect could be studied in isolation.

We started by investigating how the strength of the pyloric input influenced the gastric mill cycle period (Fig. 5). After eliminating the natural pyloric input, we introduced pyloric-like pulses into Int1 (Fig. $5 A ; n=4$ ). Incremental increases in the amplitude of these pulses decreased incrementally the period of the gastric mill rhythm, as predicted by Nadim et al. (1998) (Fig.
5). Most of the change in the gastric mill cycle period occurred during the LG interburst interval (Int1 active phase). At relatively large conductance values, we were able to return the gastric mill cycle period to the same level as occurred during the natural pyloric rhythm, when the biological AB synapse was operational (Fig. 5B). As in Figure 4, during these experiments the amplitude and duration of the disinhibitions in LG were comparable with those occurring during an ongoing pyloric rhythm.

The model had predicted that the $\mathrm{AB}$ input decreases the gastric mill cycle period via its rhythmic disinhibitions of LG. We verified this prediction by demonstrating that direct disinhibitory injections into LG also decreased the gastric mill cycle period (Fig. $6 A ; n=6$ ). Increasing the amplitude of these disinhibitions further decreased this cycle period (Fig. 6A,B). Note that the onset of each LG burst is synchronized with the pyloric-like pulses. When the dynamic-clamp pulse was large, the amplitudes of the LG disinhibitions were large compared with those of the natural pyloric rhythm. This discrepancy reflects the spatial separation of the LG soma, the LG burst generation zone, and the site of the Int1 to LG synapse.

The ability of the artificial AB synapse to decrease the gastric mill cycle period back to its value in the presence of the natural pyloric rhythm occurred consistently in all preparations [Fig. $6 C$; $n=10$; periods, $5.99 \pm 1.39 \mathrm{sec}$ (control); $20.8 \pm 7.44 \mathrm{sec}$ (no pyloric input); $6.97 \pm 1.01 \mathrm{sec}$ (reconstructed pyloric input); mean $\pm \mathrm{SD}(\sigma)]$. The gastric mill rhythm with the dynamic-clamp synapse was also quite regular, as it is during the biological pyloric 


\section{Dynamic Clamp Synapse Off}

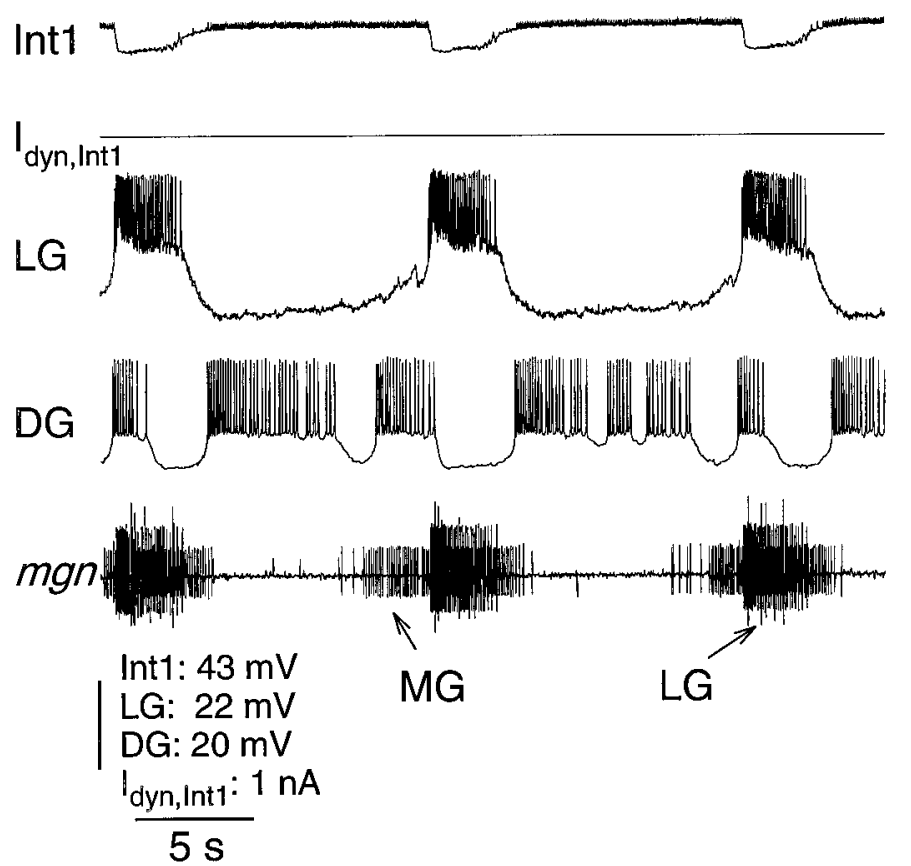

\section{Dynamic Clamp Synapse On}
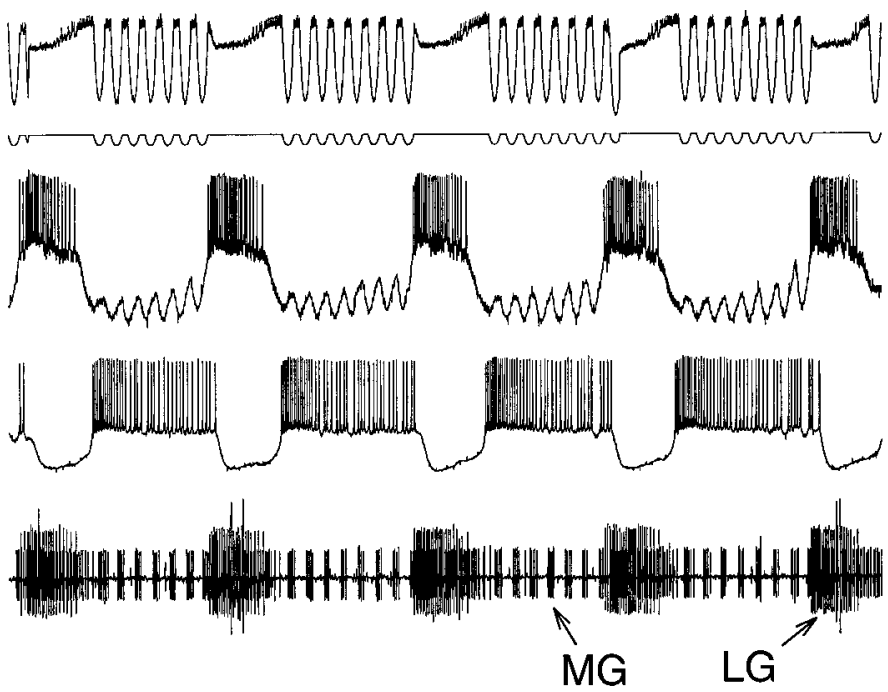

Figure 4. The artificial AB to Int1 synapse reproduces the MCN1-activated gastric mill rhythm. MCN1 was stimulated (both ions at 14 Hz each) while the natural pyloric rhythm was shut off. The resulting gastric mill rhythm exhibited a decreased cycle period when pyloric-timed dynamic-clamp IPSPs $\left(I_{d y n, \text { Int }}, g=40 \mathrm{nS}\right.$; cycle period $\left.=0.75 \mathrm{sec}\right)$ were injected into Int 1 during each $L G$ neuron interburst interval. Note the resulting rhythmic disinhibitions in $L G$, which also occur during the natural pyloric rhythm. The thickened trace at the depolarized peak of each Int1 oscillation consists of action potentials. Most hyperpolarized $\mathrm{V}_{m}$ values: $D G,-59 \mathrm{mV}$; Int $1,-55 \mathrm{mV}$; and $L G,-70 \mathrm{mV}$. dyn, Dynamic clamp; $M G$, medial gastric.

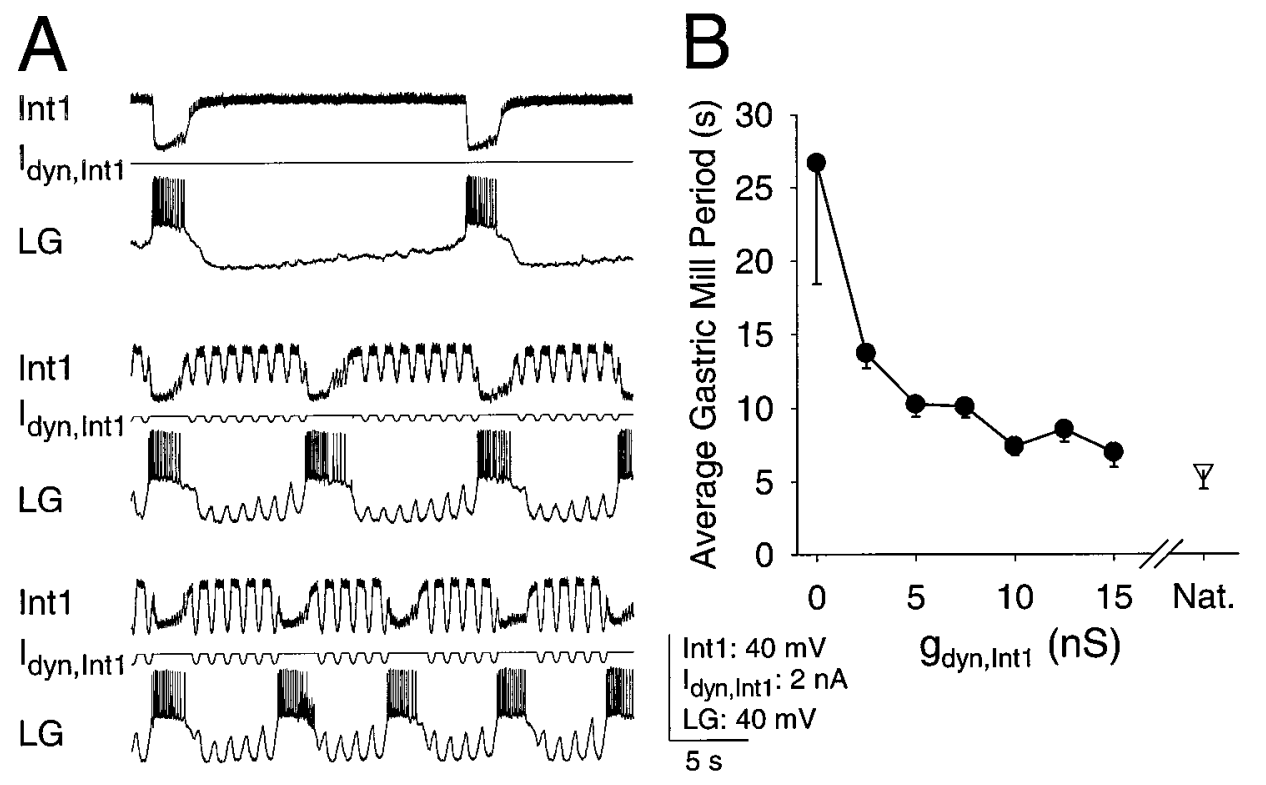

Figure 5. The gastric mill cycle period is a function of the strength of the pyloric inhibition of Int 1. A, Top, With the pyloric rhythm off, the MCN1-elicited gastric mill rhythm cycles slowly. Middle, Replacement of the $\mathrm{AB}$ inhibition of Int1 with dynamic-clamp injections into Int1 $\left(I_{d y n, \text { Int }}, g=5 \mathrm{nS}\right.$; cycle period $\left.=1 \mathrm{sec}\right)$ is sufficient to reduce the period of the MCN1-elicited gastric mill rhythm. Bottom, Increasing the strength of the dynamic-clamp injections, by increasing the conductance of the artificial synapse ( $g=10 \mathrm{nS})$, further reduces the gastric mill cycle period. Most hyperpolarized $\mathrm{V}_{m}$ values: Int $1,-43 \mathrm{mV}$; and $L G,-78 \mathrm{mV} . B$, The mean gastric mill cycle period is presented as a function of either the dynamicclamp conductance $\left(g_{\text {dyn,Int1 }}\right.$, filled circles $)$ or the cycle period during ongoing pyloric rhythms from the preparation shown in $A$ (open triangle). Each data point represents the mean $( \pm \mathrm{SD})$ gastric period from a series of cycles during a $200 \mathrm{sec}$ interval. Nat., Natural. rhythm and unlike that occurring without any pyloric input. This is reflected in the ranges of $\sigma$ for the gastric mill period in experiments in which this parameter was measured for intervals of $140 \mathrm{sec}(n=10)$ during (1) the natural pyloric rhythm (range of $\sigma, 0.34$ to 1.15 ; mean $\left._{\sigma}, 0.67\right)$, (2) no pyloric rhythm (range of $\sigma$, 0.79 to 10.31 ; $\left.\operatorname{mean}_{\sigma}, 3.49\right)$, and (3) an artificial pyloric input (range of $\sigma, 0.35$ to 1.39 ; mean $_{\sigma}, 0.82$ ).

We next examined the influence of different pyloric periods on the gastric mill cycle period. To this end, we varied the cycle period of the artificial synapse across the normal range of pyloric cycle periods $(0.5-2.0 \mathrm{sec})$, while maintaining a constant level of both MCN1 activity and dynamic-clamp synaptic conductance. In Figure $7 A$ we show the LG voltage traces when the natural pyloric rhythm was off and when artificial synapses were applied at two different pyloric periods. Note that, as in Figure $6 A$, the onset of the LG burst is synchronized with the pyloric-like pulses. Figure 
Figure 6. The gastric mill cycle period is a function of the strength of the pyloric-timed disinhibition of $L G$. $A$, The same procedure is used as in Figure $5 A$, except that dynamicclamp pulses are injected as disinhibitions into $L G$. Most hyperpolarized $\mathrm{V}_{m}: L G,-73 \mathrm{mV}$. $B$, The mean gastric mill cycle period is presented as a function of the dynamic-clamp conductance $\left(g_{\text {dyn }, L G}\right.$, filled circles $)$ and the mean gastric mill cycle period during ongoing pyloric rhythms (open triangle) from the preparation shown in $A$. Each data point represents the mean ( $\pm \mathrm{SD}$ ) gastric mill period from a series of cycles during a $200 \mathrm{sec}$ interval. $C$, Dynamic-clamp injections into $L G$ or Int1 can reproduce the gastric mill cycle period that occurs during natural pyloric rhythms. The gastric mill cycle period (mean $\pm \mathrm{SEM} ; n=10$ ) during MCN1 stimulation is plotted in the presence or absence of a natural pyloric rhythm or during dynamicclamp injections (reconstructed). The gastric mill cycle period was longer when the pyloric rhythm was off (paired $t$ test, ${ }^{* *} p<0.001$ ). There is no significant difference in the gastric mill period between the natural pyloric rhythm and the dynamic-clamp-reconstructed rhythm (paired $t$ test, $p>0.05$ ).
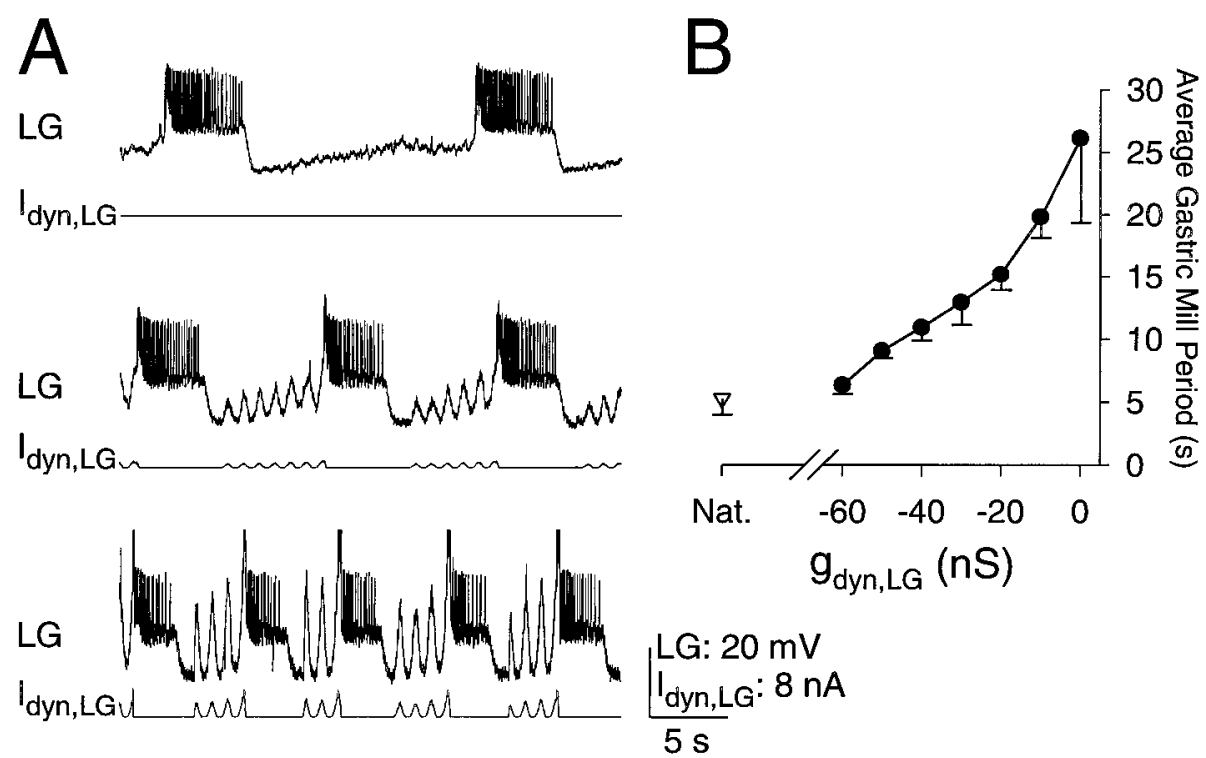

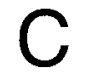

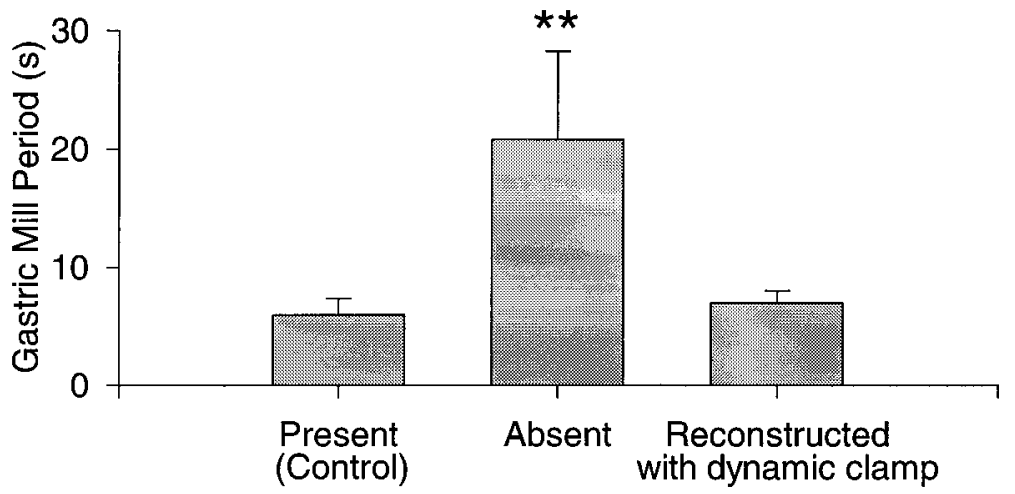

Pyloric Input
$7 B$ shows that this relationship persists across all pyloric periods (both natural and artificial). In all cases, the LG burst onset is time-locked to the onset of the preceding pyloric (or pyloric-like) burst $(n=10)$. This result concurs with predictions of the computational model (Nadim et al., 1998).

It is also evident in Figure $7 A$ that there was a faster gastric mill rhythm during the faster artificial pyloric input (period, $1 \mathrm{sec}$ ) than during the slower one (period, $2 \mathrm{sec}$ ). On average, whether the artificial input was injected into Int1 or LG, the gastric mill period increased significantly as the period of the artificial pyloric input was increased (Fig. $7 C ; n=6$; two-way ANOVA, $p<$ $0.005)$. In each experiment, the period of the artificial pyloric input was varied from 0.5 to $2.0 \mathrm{sec}$, and for each pyloric period, the mean gastric mill cycle period was determined from a continuous $140 \mathrm{sec}$ of MCN1 activation.

In this paper we define the gastric mill cycle as the interval between the onset of consecutive LG neuron bursts. As such, a direct consequence of the constant pyloric latency of the LG burst onset is that there is an integer number of pyloric cycles within a gastric mill cycle. To emphasize that each gastric mill period is an integer multiple of the corresponding pyloric period, we note that all points for the gastric mill period lie on lines of integer slope (Fig. 7D). For any value of the pyloric period, the gastric mill period commonly switched between two and three distinct values
(Fig. 7D; see also Figs. 1B, 3). If the LG burst did not occur during an expected pyloric burst (for example, because of biological variations in the network), then the LG burst would not occur until a subsequent pyloric burst. Thus, for a fixed value of pyloric cycle period there was a discrete range of gastric mill periods.

As indicated above, in general the gastric mill period increased with the pyloric period (Fig. 7C). However, unlike the case with varying the synaptic strength, there was not a strict monotonic relationship between the cycle period of the artificial AB synapse and the resulting gastric mill period. Although there was a consistent increase in gastric mill period with increases in the pyloric period (either natural or artificial), there were also points where a small increase in the pyloric period caused a decrease in the gastric mill period. For example, in Figure $7 D$ the gastric mill period dropped when the pyloric period was increased from 1.25 to $1.5 \mathrm{sec}$. In these experiments, as when the conductance level of the dynamic-clamp pulses was selectively changed while maintaining a constant frequency, most of the change in the gastric mill cycle period occurred during the LG interburst interval.

Thus far we have shown that both the MCN1-firing frequency and the rhythmic AB inhibition of Int1 contribute importantly to the regulation of the gastric mill cycle period. Both actions appear to influence gastric mill period by regulating the rate of the LG neuron escape from Int1 inhibition (Figs. 3, 5-7). However, it 


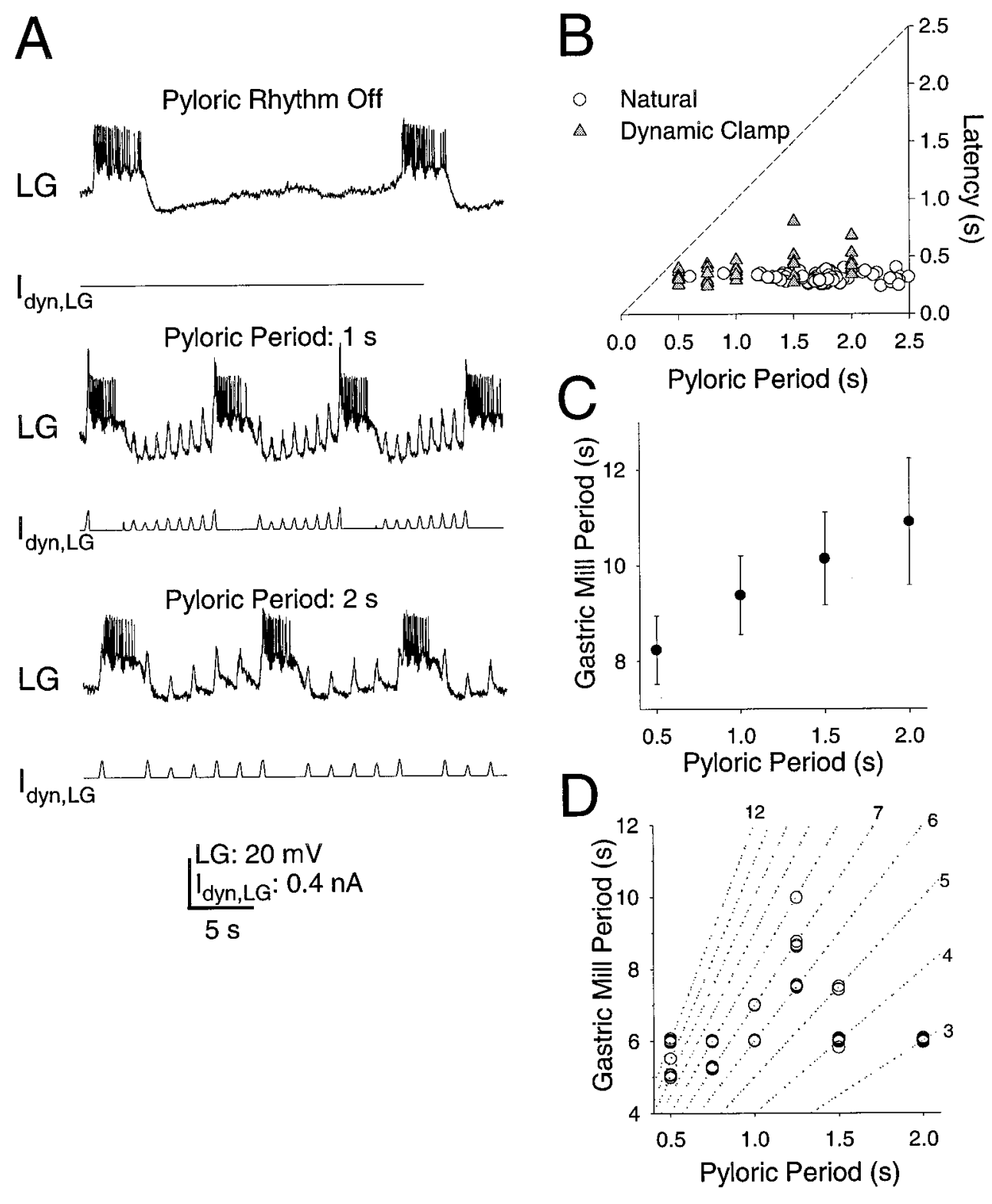

Figure 7. The gastric mill cycle period and the timing of each gastric mill cycle are a function of the frequency of the pyloric input. $A$, Top, With the pyloric rhythm turned off, the MCN1-elicited gastric mill rhythm cycles slowly. Middle, Replacement of the $\mathrm{AB}$ inhibition of Int1 with dynamic-clamp injections into $L G\left(I_{d y n, L G}\right)$ reduced the cycle period of the MCN1-elicited gastric mill rhythm. Bottom, Increasing the period of the dynamic-clamp injections caused a smaller reduction in the gastric mill cycle period. Most hyperpolarized $\mathrm{V}_{m}$ : $L G,-82 \mathrm{mV}$. $B$, Each $L G$ burst onset was time-locked to the preceding pyloric pacemaker neuron burst, both in the presence of the natural pyloric rhythm and when the natural pyloric rhythm is replaced with dynamic-clamp injections into $L G$. This relationship persists at all pyloric periods. In the case of the natural pyloric rhythm, the pyloric period was changed by injecting DC current in $\mathrm{AB}$ or $\mathrm{PD}$. The dotted line indicates the maximum possible values for the latency, where the latency is equal to the period. $C$, When the dynamic-clamp injections were made into Int 1 or $L G$, on average the gastric mill rhythm became slower as the period of the injections was increased (two-way ANOVA, $p<$ $0.005 ; n=6) . D$, There was not a strict monotonic relationship between the cycle period of the artificial AB synapse and the resulting gastric mill period within each experimental episode (140 $\mathrm{sec})$. Gastric mill periods within one episode (vertical set of data points) are plotted versus the period of the dynamic-clamp injection for that episode. Dotted lines are lines of integer slope $(y=k x$, where $k$ ranges from 3 to 12). All experimental episodes (runs of $140 \mathrm{sec}$ ) come from the same preparation. remained unclear whether the $\mathrm{AB}$ synaptic influence resulted from a cumulative influence of its periodic occurrence or whether it was a consequence of a single $\mathrm{AB}$ input occurring after the buildup of a sufficient amount of MCN1 modulatory action in LG. Note that the level to which LG is modulated by MCN1 is reset during every LG burst, because LG presynaptically inhibits MCN1 (Nusbaum et al., 1992; Coleman and Nusbaum, 1994). Our hypothesis was that there is a critical duration before the MCN1 modulation of LG grows back to a level sufficient to enable LG to generate a new burst. An indirect support for this hypothesis came from the observation that, during some disinhibitions, LG would fire only one or two action potentials (see for example Fig. $3 A$ ). Presumably, these action potentials occurred before the MCN1 modulatory actions on LG had grown enough to trigger an LG burst. We therefore examined whether a single, properly timed synaptic input would replicate the influence of rhythmic pyloric input. Here, we again eliminated the biological $\mathrm{AB}$ synapse and replaced it with dynamic-clamp pyloric-like disinhibitions into LG. Using rhythmic disinhibitory pulses in- jected into LG, under constant conditions of MCN1-firing rate and dynamic-clamp conductance, we again elicited a regular gastric mill rhythm (Fig. 8). We determined the mean LG interburst duration (Fig. 8,D) and used that value as the delay interval after each LG burst for injecting a single pyloric-like pulse of the same conductance. These single pulses were able to replicate the gastric mill cycle period that resulted from the rhythmic pyloriclike pulses. The gastric mill rhythm became entrained to these single pulses, with each disinhibitory pulse triggering an LG burst. When this same single pulse was injected at a delay $d<D$, no LG burst was elicited. Instead, the LG burst was elicited on the next pulse, which occurred at a delay $2 d>D$ (Fig. $8 ; n=8$ ).

We then determined whether this entrainment resulted from a specific interplay between the provided level of MCN1 modulation and pyloric-like synaptic strength. Consequently, we examined whether a reciprocal alteration in these two parameters could retain the same gastric mill cycle period. Indeed, when we reduced the MCN1-firing frequency but raised $g_{\text {dyn,LG }}$, single dynamic-clamp injections into LG with the delay $=D$ continued 


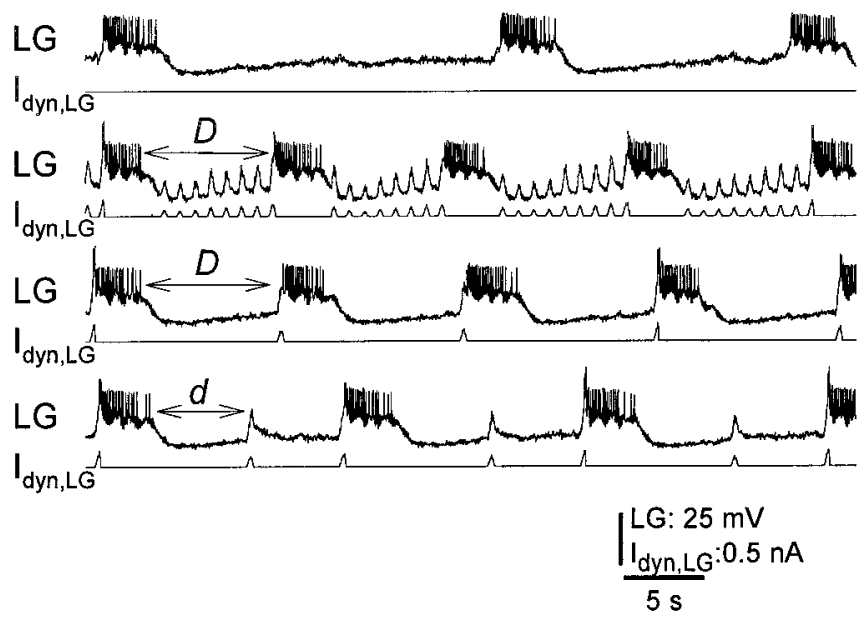

Figure 8. Single properly timed pyloric pulses are sufficient to recreate the natural gastric mill cycle period. In this experiment, the pyloric rhythm was turned off (top $L G$ trace), and pyloric-like dynamic-clamp pulses were injected into $L G\left(I_{d y n L G}, g=-50 \mathrm{nS}\right.$; cycle period $\left.=1 \mathrm{sec}\right)$. The mean $L G$ interburst duration $(D)$ during the ensuing gastric mill rhythm was determined from a sequence of cycles (second $L G$ trace from the top). This duration was used for determining the time of injection, after the end of each $L G$ burst, of a single pulse of the same conductance. When single pulses were delivered at duration $D$, each pulse elicited an $L G$ burst, and the gastric mill rhythm was entrained to these pulses (third $L G$ trace). When these single pulses were delivered at times $d<D$, no $L G$ burst was elicited until a subsequent pulse at a time $2 d>D$ (bottom $L G$ trace). Most hyperpolarized $\mathrm{V}_{m}: L G,-80 \mathrm{mV}$.

to entrain the gastric rhythm (Fig. 9, left). The gastric mill cycle period was also maintained when the MCN1-firing rate was increased while decreasing $g_{\text {dyn,LG }}$ (Fig. 9, top). Reducing either the pyloric-like synaptic strength (Fig. 9, right) or the MCN1firing rate (Fig. 9, bottom) without compensation via the other parameter eliminated the ability of the dynamic-clamp injections to entrain the gastric rhythm at the rate of the original pyloric-like input $(n=3)$. Instead, a considerably slower gastric rhythm was elicited. These data therefore indicate that both of these parameters contribute to regulation of the gastric mill cycle period. Increasing the amount of modulation to the LG neuron (by increasing the MCN1-firing frequency) and/or the strength of the AB neuron-mediated disinhibition of LG will increase the speed of the gastric mill rhythm (Fig. $10 ; n=4$ ). Additionally, increasing both parameters will elicit a faster gastric rhythm than increasing either one alone (Fig. 10).

\section{DISCUSSION}

Complex behaviors often require the coordinated interaction of distinct oscillatory neural networks that operate in distinct time domains (Dickinson, 1995). Examples of such coordination include respiration and locomotion in cats (Kawahara et al., 1989); respiration, vocalizing, and swallowing in monkeys (Larson et al., 1994); and walking and swimmeret beating in lobsters (Cattaert and Clarac, 1983). In all of these systems one central issue that remains unaddressed is the cellular and synaptic mechanisms via which distinct oscillatory networks interact to either coordinate or separate their activities.

In this study we exploited the central pattern-generating circuitry of the crab stomatogastric nervous system to explore the interaction of two rhythms that differ significantly in period. Specifically, the pyloric-timed AB neuron inhibition of Int1 allows the relatively fast pyloric rhythm to control the period of the much slower gastric mill rhythm. The AB neuron action results from its providing a "gating" signal that facilitates the transition of the LG neuron to its active state, ensuring that each transition of the LG neuron is locked to an episode of AB neuron activity. Without any input from the $\mathrm{AB}$ neuron, the $\mathrm{LG}$ neuron still reaches burst threshold, but only after a significantly longer interburst interval. By regulating the onset of the LG neuron burst via a fixed latency coupling mechanism, the $A B$ to Int1 synapse coordinates the activity of the pyloric and gastric mill rhythms.

The experimental work described here was motivated by the results of a computational model of the gastric mill rhythm elicited by the modulatory projection neuron MCN1 (Nadim et al., 1998). It is therefore gratifying that the basic insights of that model were experimentally verifiable. This was not a foregone conclusion because, although the computational model included numerous parameters that were tuned to provide an output that resembled the biological recordings, there was no assurance that this model would have accurately captured the essence of the dynamics of the biological system. The similarity of the model and experimental results argues that the mechanism revealed here is quite robust. In fact, this mechanism is likely to be a specific case of a general set of mechanisms in which a slow process in a neuron builds toward an asymptote (e.g., burst generation) and a phasic faster process can facilitate reaching that end point. This same general result could be achieved with synaptic and cellular details other than those described here with essentially the same results.

Coupling between oscillatory circuits underlying locomotion has been extensively studied, both theoretically and experimentally. For example, in vertebrates that swim with undulatory trunk movements, the underlying neuronal circuitry is iterated in each segment of the spinal cord (Grillner et al., 1995). The study of chains of segmental oscillators has led to a comprehensive mathematical theory of phase-coupled oscillators (Marder et al., 1997b; Sigvardt and Miller, 1998). This theory predicts a relationship between intrinsic frequencies and the resulting phase difference between oscillators in the chain. In particular, the phase difference between the oscillators is dependent on the strength of the coupling between them, but for fixed coupling strength, it remains constant with changes in frequency. However, this mathematical theory deals only with coupled oscillators that are identical or close in frequency and whose dynamics can be expressed in terms of their relative phase. The model proposed by Nadim et al. (1998) and experimentally verified in this study is novel in that it is based on a latency-locking mechanism between two oscillators with widely different frequencies. In this mechanism the latency, not the phase, between the two oscillators is fixed. Consequently, as the frequency increases, the phase difference (calculated with respect to the frequency of either oscillator) gradually increases.

The results reported in this paper hold for the MCN1-activated gastric mill rhythm. This variant of the gastric mill rhythm is activated by the MCN1 projection neuron, which acts in part by slowly depolarizing the LG neuron during its interburst interval. As described in this paper, this slow depolarization enables the pyloric input to exert its influence on the gastric mill rhythm and hence to coordinate the activity of the two rhythms. It is important to stress that the gastric mill rhythm may be activated by other projection neurons (Norris et al., 1994) or substances (Weimann et al., 1993; Weimann and Marder, 1994) that render different functional network configurations. In these gastric mill rhythms, we would predict that the pyloric influence on the gastric 


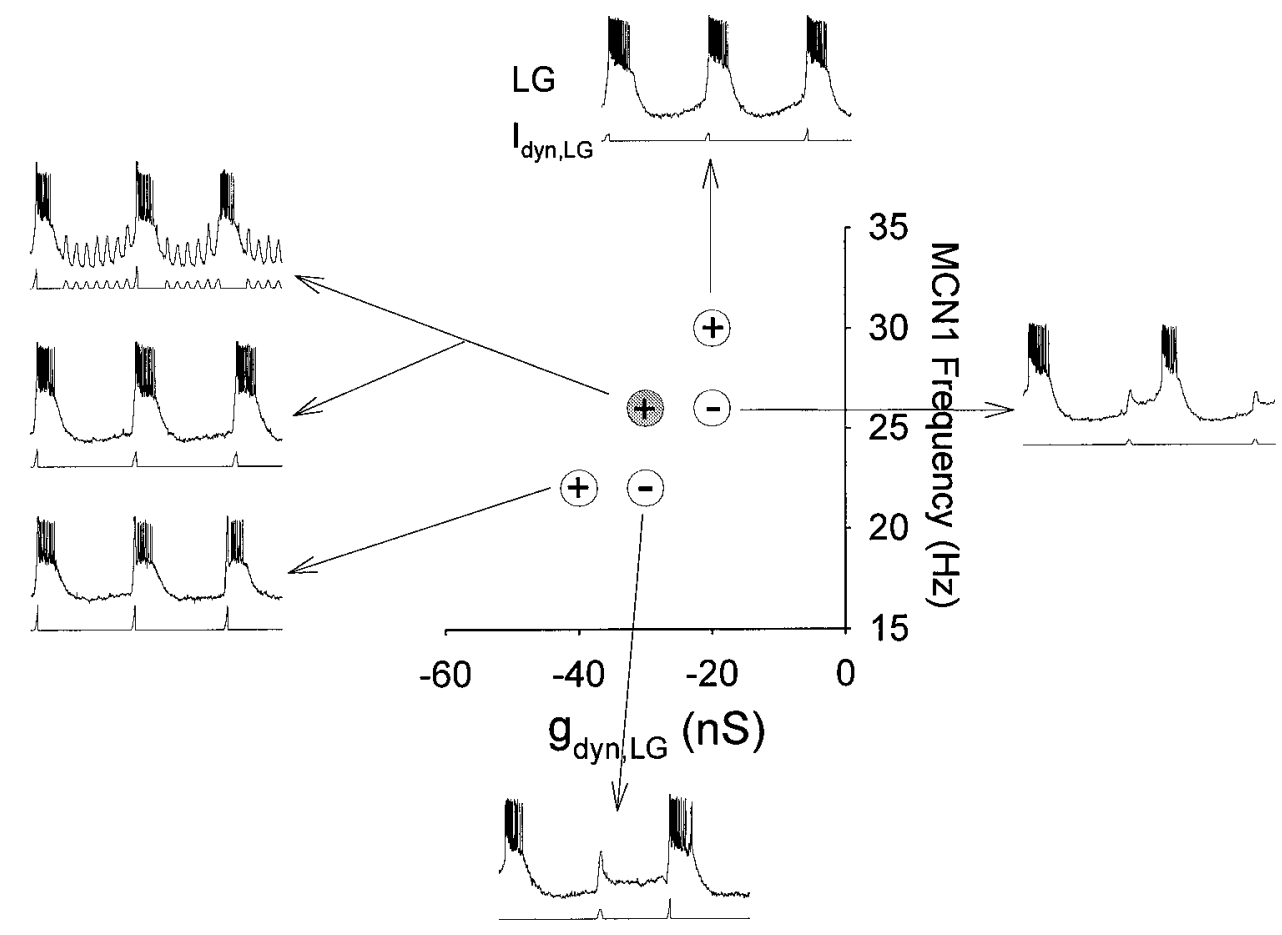

Figure 9. The gastric mill cycle period is a function of both the MCN1-firing frequency and the strength of the rhythmic input from the $\mathrm{AB}$ neuron. Left (top), Pyloric-like dynamic-clamp pulses $\left(g_{d y n, L G}=-30 \mathrm{nS}\right)$ were injected into $L G$ during $M C N 1$ stimulation $(25 \mathrm{~Hz})$ to elicit the gastric mill rhythm. Left (middle), As in Figure 8, replacement of the rhythmic pulses with single pulses at duration $D$ replicated the gastric mill cycle period. Left (bottom), Top, Single pulses were also able to replicate this cycle period if either parameter were reduced while the other parameter was sufficiently increased. Right, Bottom, If either parameter were reduced while the other one was maintained constant, single pulses did not elicit an $L G$ burst. + indicates single dynamicclamp pulses that entrained the gastric mill rhythm to the period resulting from rhythmic pulses; + with shaded circle indicates entrainment with control parameters; - indicates no such entrainment.

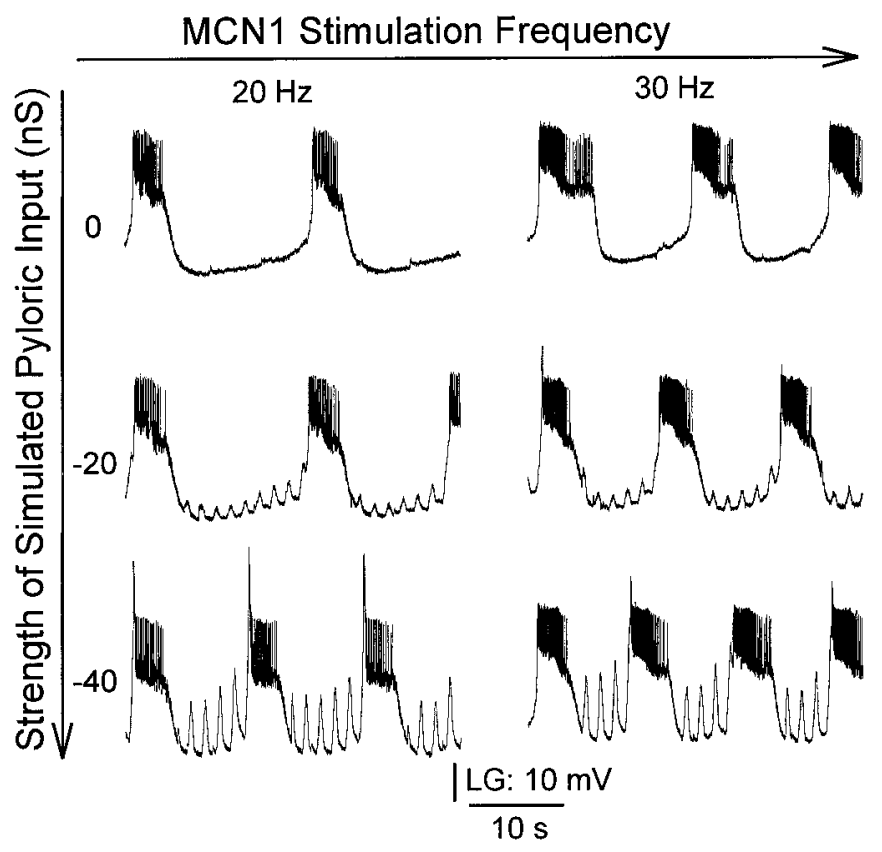

Figure 10. The gastric mill cycle period is a function of both the MCN1-firing frequency and the strength of the pyloric-timed input. Increasing either parameter speeds up the gastric mill rhythm, and increasing both parameters is more effective than increasing either one alone. Data shown are from a single preparation.

mill period will be altered or absent. Therefore, activation of different forms of the gastric mill rhythm by different input neurons is likely to determine the extent and direction of the pyloric influence on the gastric mill rhythm. One important implication is that neuromodulation provides flexibility not only to individual central pattern-generating circuits (Marder and Calabrese, 1996) but also to intercircuit interactions.
In a related study, Bartos and Nusbaum (1997a) showed that during MCN1 activity the gastric mill rhythm also influences the pyloric rhythm. Comparable gastric mill influences on the pyloric rhythm occur also in the lobster stomatogastric system (Clemens et al., 1998). There is good reason for the pyloric and gastric mill rhythms to be coordinated, at least under some sets of physiological conditions, because they control related aspects of the feeding process in crustaceans. The gastric mill system causes the rhythmic contraction of muscles that move the teeth in the gastric mill compartment of the crustacean stomach, thereby controlling the chewing of food (Johnson and Hooper, 1992). The pyloric system controls the rhythmic contraction of dilator and constrictor muscles in the pyloric compartment of the stomach, thereby controlling the pumping and filtering of chewed food from the gastric mill to the midgut. Surprisingly, our data suggest that the pyloric rhythm not only contributes to regulation of the gastric mill cycle period but, under some conditions, is necessary for the normal operation of the gastric mill rhythm. The normal range of gastric mill cycle periods in the crab extends from 5 to $15 \mathrm{sec}$, both in vitro and in vivo (Heinzel et al., 1993; Norris et al., 1994). The mean gastric mill rhythm that occurred in the absence of the pyloric rhythm ( $\sim 20 \mathrm{sec}$; see Fig. 2$)$ was slower than this normal range.

Previous work in the crab STG showed that several STG neurons are rhythmically active with both the gastric mill and pyloric rhythms and that they participate actively in the generation of both rhythms (Weimann et al., 1991; Weimann and Marder, 1994). This, in combination with the ability of different neuromodulators to elicit different forms of the gastric mill and pyloric rhythms (Marder and Calabrese, 1996), argues that there is a single STG network from which are selected different statedependent functional circuits. The idea of nested circuits within a larger network ensemble is reinforced by the present discovery that the pyloric rhythm is necessary for generation of an appropriate gastric mill cycle period.

It is now well established that both neuromodulators and the 
intrinsic dynamics of a network can alter synaptic strength (Johnson and Harris-Warrick, 1990; Manor et al., 1997). These alterations provide the possibility for regulating the period of a rhythmically active circuit at multiple sites, both within and external to that circuit. Our work demonstrates that a neuromodulatory action on one neuronal circuit could indeed have functional consequences on a second circuit with which it interacts synaptically. The mechanism demonstrated in the current study provides a route through which such indirect functional influences on a neuronal circuit can arise.

\section{REFERENCES}

Bartos M, Nusbaum MP (1997a) Intercircuit control of motor pattern modulation by presynaptic inhibition. J Neurosci 17:2247-2256.

Bartos M, Nusbaum MP (1997b) Frequency regulation of a slow rhythm by periodic inputs from a fast rhythm in the crab stomatogastric ganglion. Soc Neurosci Abstr 23:476.

Cattaert D, Clarac F (1983) Influence of walking on swimmeret beating in the lobster Homarus gammarus. J Neurobiol 14:421-439.

Clemens S, Massabuau JC, Legeay A, Meyrand P, Simmers J (1998) In vivo modulation of interacting central pattern generators in lobster stomatogastric ganglion: influence of feeding and partial pressure of oxygen. J Neurosci 18:2788-2799.

Coleman MJ, Nusbaum MP (1994) Functional consequences of compartmentalization of synaptic input. J Neurosci 14:6544-6552.

Coleman MJ, Nusbaum MP, Cournil I, Claiborne BJ (1992) Distribution of modulatory inputs to the stomatogastric ganglion of the crab, Cancer borealis. J Comp Neurol 325:581-594.

Coleman MJ, Meyrand P, Nusbaum MP (1995) A switch between two modes of synaptic transmission mediated by presynaptic inhibition. Nature 378:502-505.

Dickinson PS (1995) Interactions among neural networks for behavior. Curr Opin Neurobiol 5:792-798.

Graubard K, Raper JA, Hartline DK (1980) Graded synaptic transmission between spiking neurons. Proc Natl Acad Sci USA 77:3733-3735.

Gray CM (1995) Synchronous oscillations in neuronal systems: mechanisms and functions. J Comput Neurosci 1:11-38.

Grillner S, Deliagina T, Ekeberg O, El Manira A, Hill RA, Lansner A, Orlovsky GN, Wallen P (1995) Neural networks that coordinate locomotion and body orientation in lamprey. Trends Neurosci 18:270-279.

Harris-Warrick RM, Marder E, Selverston AI, Moulins M (1992) Dynamic biological networks: the stomatogastric nervous system. Cambridge, MA: MIT.

Heinzel H-G, Weimann JM, Marder E (1993) The behavioral repertoire of the gastric mill in the crab Cancer pagurus: an in situ endoscopic and electrophysiological examination. J Neurosci 13:1793-1803.

Johnson BR, Harris-Warrick RM (1990) Aminergic modulation of graded synaptic transmission in the lobster stomatogastric ganglion. J Neurosci 10:2066-2076.

Johnson BR, Hooper SL (1992) Overview of the stomatogastric nervous system. In: Dynamic biological networks: the stomatogastric nervous system (Harris-Warrick RM, Marder E, Selverston AI, Moulins M, eds), pp 1-30. Cambridge, MA: MIT.

Kawahara K, Kumagai S, Nakazono Y, Myamoto Y (1989) Coupling between respiratory and stepping rhythms during locomotion in decerebrate cats. J Appl Physiol 67:110-115.

Larson CR, Yajima Y, Ko P (1994) Modification in activity of medullary respiratory-related neurons for vocalizing and swallowing. J Neurophysiol 71:2294-2304.
Laurent G (1996) Dynamical representation of odors by oscillating and evolving neural assemblies. Trends Neurosci 19:489-496.

Laurent G (1997) Olfactory processing: maps, time and codes. Curr Opin Neurobiol 7:547-553.

Lisman JE (1997) Bursts as a unit of neural information: making unreliable synapses reliable. Trends Neurosci 20:38-43.

Manor Y, Nadim F (1997) A program for simulating synaptic activity using LabWindows/CVI, pp 207-215. Houston: National Instruments.

Manor Y, Nadim F, Abbott LF, Marder E (1997) Temporal dynamics of graded synaptic transmission in the lobster stomatogastric ganglion. J Neurosci 17:5610-5621.

Manor Y, Nadim F, Bartos M, Marder E, Nusbaum MP (1998) Dynamic regulation of motor pattern generation via disinhibition and neuromodulation. Soc Neurosci Abstr 24:1892.

Marder E, Calabrese RL (1996) Principles of rhythmic motor pattern generation. Physiol Rev 76:687-717.

Marder E, Jorge-Rivera JC, Kilman V, Weimann JM (1997a) Peptidergic modulation of synaptic transmission in a rhythmic motor system. Adv Organ Biol 2:213-233.

Marder E, Kopell N, Sigvardt K (1997b) How computation aids in understanding biological networks. In: Neurons, networks, and motor behavior (Stein PSG, Grillner S, Selverston AI, Stuart DG, eds), pp 139-149. Cambridge, MA: MIT.

McCormick DA, Bal T (1997) Sleep and arousal: thalamocortical mechanisms. Annu Rev Neurosci 20:185-215.

Nadim F, Manor Y, Nusbaum MP, Marder E (1998) Frequency regulation of a slow rhythm by a fast periodic input. J Neurosci 18:5053-5067.

Norris BJ, Coleman MJ, Nusbaum MP (1994) Recruitment of a projection neuron determines gastric mill motor pattern selection in the stomatogastric nervous system of the crab, Cancer borealis. J Neurophysiol 72:1451-1463.

Norris BJ, Coleman MJ, Nusbaum MP (1996) Pyloric motor pattern modification by a newly identified projection neuron in the crab stomatogastric nervous system. J Neurophysiol 75:97-108.

Nusbaum MP, Weimann JM, Golowasch J, Marder E (1992) Presynaptic control of modulatory fibers by their neural network targets. J Neurosci 12:2706-2714.

Sharp AA, O'Neil MB, Abbott LF, Marder E (1993a) Dynamic clamp: computer-generated conductances in real neurons. J Neurophysiol 69:992-995.

Sharp AA, O'Neil MB, Abbott LF, Marder E (1993b) The dynamic clamp: artificial conductances in biological neurons. Trends Neurosci 16:389-394.

Sigvardt KA, Miller WL (1998) Analysis and modeling of the locomotor central pattern generator as a network of coupled oscillators. In: Neuronal mechanisms for generating locomotor activity (Kiehn O, HarrisWarrick RM, Jordan LM, Hultborn H, Kudo N, eds), pp 250-265. New York: NY Academy of Sciences.

Skinner FK, Mulloney B (1998) Intersegmental coordination in invertebrates and vertebrates. Curr Opin Neurobiol 8:725-732.

Weimann JM, Marder E (1994) Switching neurons are integral members of multiple oscillatory networks. Curr Biol 4:896-902.

Weimann JM, Meyrand P, Marder E (1991) Neurons that form multiple pattern generators: identification and multiple activity patterns of gastric/pyloric neurons in the crab stomatogastric system. J Neurophysiol 65:111-122.

Weimann JM, Marder E, Evans B, Calabrese RL (1993) The effects of SDRNFLRFamide and TNRNFLRFamide on the motor patterns of the stomatogastric ganglion of the crab Cancer borealis. J Exp Biol 181:1-26. 